\title{
Molecular cytogenetic characterization of a novel wheat-Psathyrostachys huashanica Keng T3DS-5NsL-5NsS and T5DL-3DS·3DL dual translocation line with powdery mildew resistance
}

Jiachuang Li', Li Zhao', Xueni Cheng², Guihua Bai ${ }^{3}$, Mao Li', Jun Wu', Qunhui Yang ${ }^{1}$, Xinhong Chen', Zujun Yang ${ }^{4}$ and Jixin Zhao ${ }^{1 *}$

\begin{abstract}
Background: Psathyrostachys huashanica Keng ( $2 n=2 x=14$, NsNs) carries many outstanding agronomic traits, therefore is a valuable resource for wheat genetic improvement. Wheat-P. huashanica translocation lines are important intermediate materials for wheat breeding and studying the functions of alien chromosomes. However, powdery mildew resistance in these translocation lines has not been reported previously.

Results: This study developed a novel wheat-P. huashanica translocation line TR77 by selecting a $F_{7}$ progeny from the cross between heptaploid hybrid H8911 ( $2 n=7 x=49$, AABBDDNs) and durum wheat line Trs-372. Chromosome karyotype of $2 n=42=21 \|$ was observed in both mitotic and meiotic stages of TR77. Genomic in situ hybridization analysis identified two translocated chromosomes that paired normally at meiosis stage in TR77. Molecular marker analysis showed that part of chromosome 5D was replaced by part of alien chromosome fragment $5 \mathrm{Ns}$. It meant replacement made part 5DL and part 5NsL.5NsS existed in wheat background, and then translocation happened between these chromosomes and wheat 3D chromosome. Fluorescence in situ hybridization demonstrated that TR77 carries dual translocations: T3DS-5NsL·5NsS and T5DL-3DS·3DL. Analysis using a $15 \mathrm{~K}$-wheat-SNP chip confirmed that SNP genotypes on the 5D chromosome of TR77 matched well with these of $P$. huashanica, but poorly with common wheat line 7182. The translocation was physically located between 202.3 and $213.1 \mathrm{Mb}$ in 5D. TR77 showed longer spikes, more kernels per spike, and much better powdery mildew resistance than its wheat parents: common wheat line 7182 and durum wheat line Trs-372.

(Continued on next page)
\end{abstract}

\footnotetext{
* Correspondence: zhjx881@163.com

'Shaanxi Key Laboratory of Plant Genetic Engineering Breeding, College of Agronomy, Northwest A\&F University, Yangling 712100, Shaanxi, China

Full list of author information is available at the end of the article
}

(C) The Author(s). 2020 Open Access This article is licensed under a Creative Commons Attribution 4.0 International License, which permits use, sharing, adaptation, distribution and reproduction in any medium or format, as long as you give appropriate credit to the original author(s) and the source, provide a link to the Creative Commons licence, and indicate if changes were made. The images or other third party material in this article are included in the article's Creative Commons licence, unless indicated otherwise in a credit line to the material. If material is not included in the article's Creative Commons licence and your intended use is not permitted by statutory regulation or exceeds the permitted use, you will need to obtain permission directly from the copyright holder. To view a copy of this licence, visit http://creativecommons.org/licenses/by/4.0/ The Creative Commons Public Domain Dedication waiver (http://creativecommons.org/publicdomain/zero/1.0/) applies to the data made available in this article, unless otherwise stated in a credit line to the data. 
(Continued from previous page)

Conclusions: TR77 is a novel stable wheat-P. huashanica T3DS-5NsL.5NsS and T5DL-3DS·3DL dual translocation line and showed significant improved spike traits and resistance to powdery mildew compared to its parents, thus, it can be an useful germplasm for breeding disease resistance and studying the genetic mechanism of dual translocations.

Keywords: In situ hybridization, Dual translocation line, Psathyrostachys huashanica, Single nucleotide polymorphism array, Wheat powdery mildew

\section{Background}

Wheat (Triticum aestivum $\mathrm{L}, 2 n=42$, AABBDD) is one of the most widely cultivated crops throughout the world. Also, wheat yield can be influenced by heat, drought, insects and diseases, while relatively narrow genetic variation in wheat makes it even more difficulty to identify sources of resistance to combat these biotic and abiotic stresses [1,2]. Wheat wild relatives, however, carry many beneficial genes that can be used to improve wheat resistance to these stresses as well as grain yield and quality [3]. For example, a wheat-Agropyron 6P chromosome disomic addition line has more kernels per spike than its wheat parent [4]; a wheat-Aegilops substitution line called $\mathrm{PRH}_{5}$ showed high iron and zinc content [5]; a wheatHaynaldia villosa 4DL-4VS translocation line called NAU413 exhibited high resistance to wheat spindle streak mosaic virus [6]; and wheat-rye $1 \mathrm{BL} / 1 \mathrm{RS}$ translocation lines have been widely used in wheat breeding to improve resistance to multiple diseases and abiotic stress [3, 7].

Psathyrostachys huashanica Keng $(2 n=2 x=14$, NsNs) is an endangered species of Poaceae, Triticeae found only in high mountains of China. As a wild relative of wheat, it owns many outstanding traits, such as high tolerance to abiotic stress, i.e. salinity, alkalinity, cold and drought and high resistance to normal wheat diseases, i.e. rust, take-all and powdery mildew [8-11]. Our laboratory made a wide cross between $P$. huashanica and common wheat line 7182 in the 1980s and obtained the F1 hybrid, H811 ( $2 n=28$, ABDNs), using in vitro culture of young embryos. The colchicine treated H811 was crossed and backcrossed to 7182 to get heptaploid hybrid H8911 ( $2 n=49$, AABBDDNs). Subsequently, H8911 was then backcrossed to 7182 or crossed to several other wheat cultivars, and generated a series of wheat-P. huashanica derived lines including wheat $-P$. huashanica $1 \mathrm{Ns}-7 \mathrm{Ns}$ disomic addition lines [12-17], 2Ns(2D) disomic substitution line [18], $5 \mathrm{Ns}(5 \mathrm{D})$ disomic substitution line [19], and translocation lines [20, 21]. These wheat- $P$. huashanica derived lines have more desired agronomic traits than their wheat parents, demonstrating that $P$. huashanica is a useful wild relative for wheat improvement. However, all the known wheat-P. huashanica translocation lines are poorly characterized [20-23].
Although previous studies identified the transposition of wheat and $P$. huashanica chromosomes in some wheat$P$. huashanica derived lines, stability of these alien chromosomes in wheat, their homeologous relationship to wheat chromosomes, and actual translocation sites in wheat chromosomes remain to be investigated. Therefore, determining chromosomal composition of stable wheat- $P$. huashanica translocation lines may facilitate applications of this type of resources in wheat breeding.

Disease resistance genes from wild relatives of wheat play important roles in control of powdery mildew. Among the 25 wheat closely related genera, 14 demonstrated immunity to powdery mildew, including Secale [24], Aegilops [25], Thinopyrum [26], and Haynaldia villosa [27]. P. huashanica is also immune to powdery mildew but wheat-P. huashanica derived lines with powdery mildew resistance have not been reported to date.

In this study, we developed a novel wheat-P. huashanica translocation line with resistance to powdery mildew from a $F_{7}$ progeny of wheat- $P$. huashanica heptaploid line H8911 × durum wheat (Triticum durum L, $2 n=28$, AABB) line Trs-372. The objectives of this study were to: (a) determine the pairing and inherent stability of the transposed alien chromosome segment using cytogenetic methods; (b) examine the chromosomal composition of the derived line using molecular markers and fluorescence in situ hybridization (FISH); (c) determine the physical locations of the translocation sites on the chromosomes using a wheat $15 \mathrm{~K}$ single nucleotide polymorphism (SNP) array; and (d) evaluate the agronomic traits of the new line to predict its potential use in wheat breeding.

\section{Results}

\section{Mitotic cells of TR77}

About 98\% of metaphase mitotic root tip cells (RTCs) of TR77 had a chromosome number of $2 n=42$ (Fig. 1a, Table 1) among 136 microscopically screened mitotic RTCs that were randomly selected from different plants. All the chromosomes were clearly separated from each other in metaphase. Genomic in situ hybridization (GISH) analysis using the whole genomic DNA from $P$. huashanica as the probe and wheat 7182 as the block on the same slide 


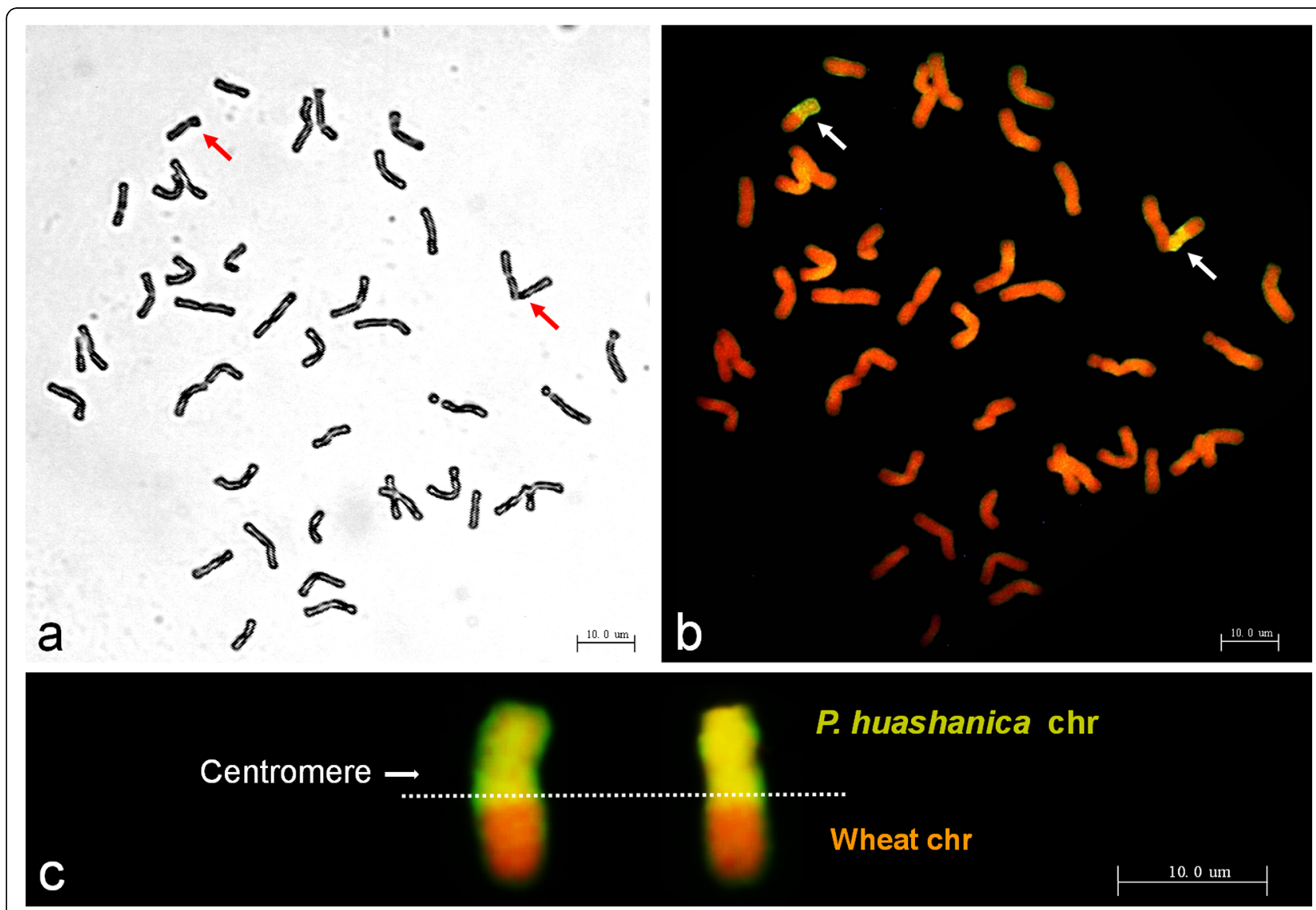

Fig. 1 Cytological and genomic in situ hybridization (GISH) analyses of root tip cells from line TR77. a Mitotic metaphase, $2 n=42$. b GISH analysis of TR77. GISH was conducted using the total DNA from P. huashanica as the probe and 7182 as the block. Two chromosomal segments with fluorescent hybridization signals (yellow-green) were detected as alien chromosomes in the mitotic metaphase. c Enlarged a pair of the translocated chromosomes. The alien chromosome segment consists of the short arm and a small portion of the long arm attached to the centromere. Chromosomes were counterstained with Propidium lodide (red)

identified a pair of chromosomal segments with strong yellow-green hybridization signals (Fig. 1b). Each of the signals was emitted from nearly half of the wheat chromosome, and clearly covered the entire short arm and partial long arm of the chromosome that were connected through the centromere (Fig. 1c), indicating the wheat chromosomes were evidently transposed with $P$. huashanica chromosomal segments. Therefore, TR77 was a wheat $-P$. huashanica line with a large segment translocation.

\section{Chromosomes in meiosis cells of TR77}

Pollen mother cells (PMCs) in metaphase I of TR77 had a chromosome configuration of $2 n=21 \mathrm{II}$ (Table 1, Fig. 2a).
In meiosis anaphase I, trivalents or quadrivalents and lagging chromosomes were not observed (Fig. 2b). GISH analysis on PMCs in the important fission periods detected one tetrad with a semicircular fluorescent signal in meiosis prophase I (Fig. 2c), and then a rod chromosome with a partial yellow-green signal appeared when the chromosomes were arranged on the equatorial plate (Fig. 2d), separated into two chromosomes, and finally moved to the two poles of the cell in meiotic anaphase I (Fig. 2e). In meiosis telophase II, each of the four sperms carried a fluorescent segment (Fig. 2f). The pair of transposed $P$. huashanica chromosome fragments showed normal synapsis, pairing, segregation, and inheritance in the wheat

Table 1 Chromosome numbers and pairing status in the meiotic and meiotic phases of TR77

\begin{tabular}{|c|c|c|c|c|c|c|c|c|}
\hline \multirow[t]{3}{*}{ Material } & \multirow{3}{*}{$\begin{array}{l}\text { No. of counted } \\
\text { cells }\end{array}$} & \multirow[t]{3}{*}{$2 n$} & \multicolumn{6}{|c|}{ Chromosome configuration } \\
\hline & & & \multirow[t]{2}{*}{ Univalent } & \multicolumn{3}{|l|}{ Bivalent } & \multirow[t]{2}{*}{ Trivalent } & \multirow[t]{2}{*}{ Quadrivalent } \\
\hline & & & & Rod & Ring & Total & & \\
\hline TR77 & 128 & 42 & $0.12(0-2)$ & $1.31(1-3)$ & $19.54(19-21)$ & $20.85(20-21)$ & 0 & 0 \\
\hline
\end{tabular}




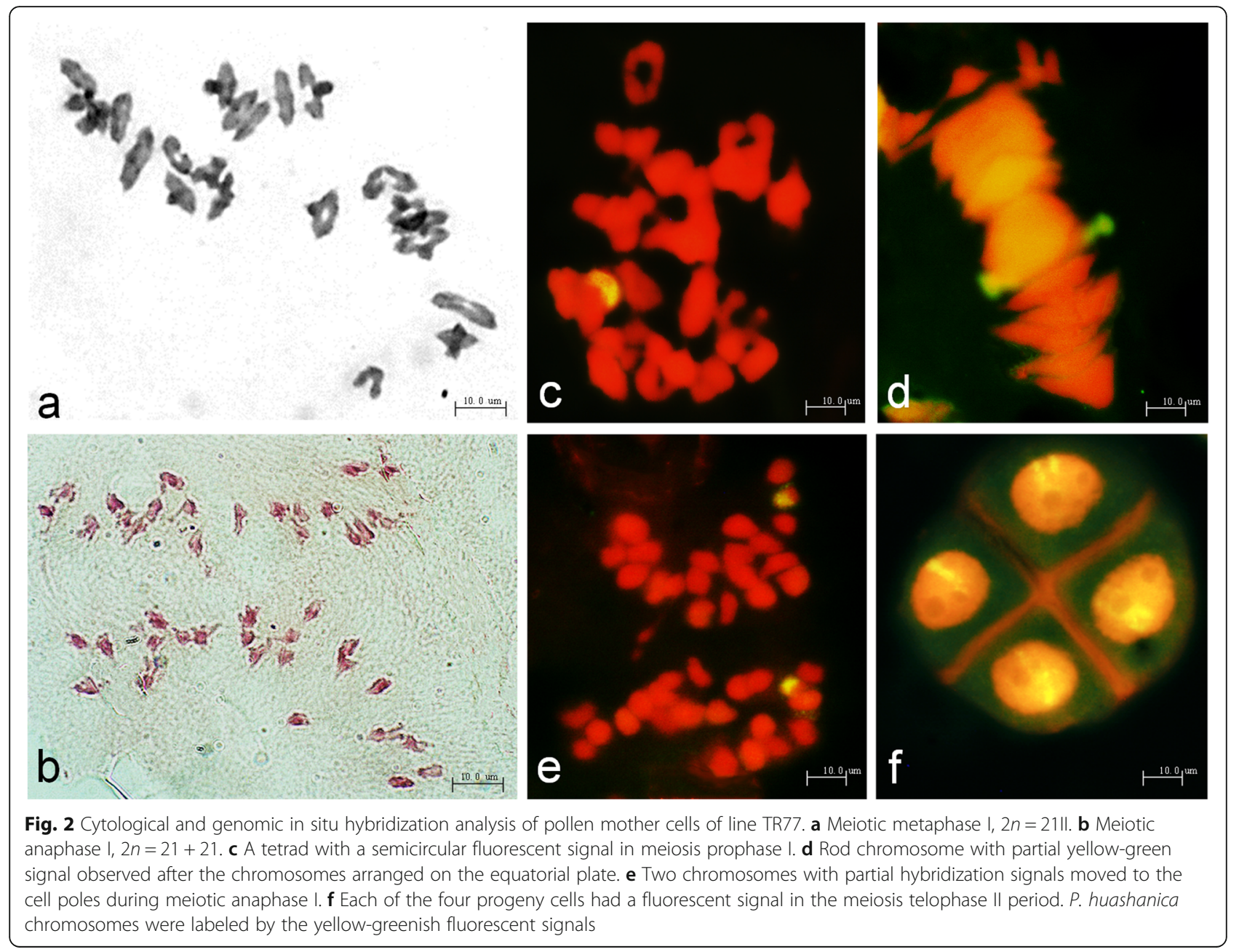

background, therefore, they came from the same homoeologous groups, which confirmed that TR77 was a cytogenetically stable wheat $-P$. huashanica translocation line.

\section{Analysis of molecular markers in TR77}

We screened 384 pairs of simple sequence repeat (SSR) primers distributed among the long and short arms of the wheat chromosomes to determine the homoeologous groups for the transposed wheat chromosomes in TR77 (Table S1, Figure S1). Except for 5D that have only a few markers amplified on 5DL of TR77, the markers on other wheat genomes amplified the same wheat chromosome specific bands between the wheat parent line 7182 and TR77, therefore TR77 might loss its whole short arm and part of the long arm of 5D chromosome. Further comparison of the SSR markers on the wheat 5D chromosome (Table 2, Fig. 3) between 7182 and TR77 found that the primers from 5DS and part of 5DL amplified wheat D genome-specific bands in the parent 7182 , but not in TR77, confirming that the short arm and part of the long arm of the 5D chromosome were missing in the TR77, and the translocation position might be near the marker $X c f d 8$ because the marker Xgdm153 close to $X c f d 8$ did not amplify in TR77.

Among the 78 pairs of expressed sequence tag-sequence tagged site (EST-STS) markers from seven wheat homoeologous groups, three pairs of primers, BF146187, $B E 444644$ and $C D 452608$, from the wheat homoeologous group 5 (5AS, 5BS, and 5DS) (Table 2, Figure S1 and Fig. 4) amplified in P. huashanica and TR77, but not in wheat line 7182, suggesting that the three markers are Ns genome-specific and alien chromosome segment in line TR77 was from $P$. huashanica $5 \mathrm{Ns}$ chromosome.

\section{FISH analysis of TR77}

FISH analysis was performed to determine the transposed wheat chromosomes using repetitive oligonucleotide sequences pSc119.2 and pTa535 as probes. FISH results of line TR77, common wheat 7182 and P. huashanica could be seen in Fig. 5a, b and c, respectively. All the chromosomes of TR77 showed the same fluorescent karyotype as wheat line 
Table 2 Wheat SSR and EST-STS markers used in this study to analyze the chromosomal composition of TR77

\begin{tabular}{|c|c|c|c|c|c|c|c|}
\hline markers & locus & 7182 & Trs-372 & TR77 & $P$ & $\begin{array}{l}\text { Primer } \\
\left(5^{\prime}-3^{\prime}\right)\end{array}$ & $\operatorname{Tm}\left({ }^{\circ} \mathrm{C}\right.$ \\
\hline Barc130 & 5DS & + & - & - & & F: CGGCTAGTAGTTGGAGTGTTGG R: ACCGCCTCTAGTTATTGCTCTC & 50 \\
\hline Xсfd189 & 5DS & + & - & - & & F: GCTAAAGCCACATAGGACGG R: GCACAAGATTTGCAAGGCT & 60 \\
\hline Xwmc318 & $5 \mathrm{DL}$ & + & - & - & & F: CGTAAAATTACGGTGCATTGAT R: GTGGACTTITGTGGTTITGAG & 60 \\
\hline Xgdm153 & $5 \mathrm{DL}$ & + & - & - & & F: TATAGGCAAATTAATTAAGACG R: ATCTTTATGTGAGTACACTGC & 50 \\
\hline Xcfd8 & $5 \mathrm{DL}$ & + & - & + & & F: ACCACCGTCATGTCACTGAG R: GTGAAGACGACAAGACGCAA & 60 \\
\hline Xgwm182 & $5 \mathrm{DL}$ & + & - & + & & F: TGATGTAGTGAGCCCATAGGC R: TTGCACACAGCCAAATAAGG & 60 \\
\hline Xwmc357 & $5 \mathrm{DL}$ & + & - & + & & F: TAGTGGGTGACCGGTCAAGA R: TGGACGGATTTGGTCATTC & 60 \\
\hline BF146187 & 5AS 5BS 5DS & - & - & + & + & F: CAAGGTGCAACAGTTCATGG R: GGTCACAGAAATATGCGGCT & 60 \\
\hline BE444644 & 5AS 5BS 5DS & - & - & + & + & F: AAGCTTGCTGAGCTTTCTGG R: TTGAGGGATGTAGGGCAAAG & 60 \\
\hline CD452608 & 5AS 5BS 5DS & - & - & + & + & F: TGATGTCTTGTCGTGGTCGT R: TITGGATGCAGCAAGACAG & 60 \\
\hline
\end{tabular}

$P$ indicates $P$. huashanica; + means marker is present; - means marker is absent

$7182[28,29]$, except for the chromosomes 3D and 5D (Fig. $5 \mathrm{~d})$ that showed translocations. One breakage site was observed between the long arm of 5D chromosome and short arm of 3D chromosomes of wheat (Fig. 5e), which was caused by loss of most portion wheat $5 \mathrm{D}$ chromosome and that remaining 5DL segment was combined with most portion of chromosome 3D (3DS.3DL) to become 5DL3DS.3DL. The other breakage site was observed between the wheat 3DS chromosome and most portion of newly acquired $P$. huashanica chromosome $5 \mathrm{Ns}(\mathrm{NsL} \cdot \mathrm{NsS})$ segment (Fig. 5e) due to reciprocal translocations and recombination between remaining 3DS segment and the 5Ns segment (NsL.NsS) of $P$. huashanica to form a new translocation chromosome 3DS-NsL.NsS. Thus, TR77 was a translocation line with dual translocations.

\section{Comparison of fingerprints of TR77 and its parents using a SNP Array}

To determine the chromosomal composition of TR77, wheat $15 \mathrm{~K}$ SNP arrays were employed to genotype TR77and its parents (Table S2). In general, significantly higher percentages of common SNP sequences on all 21 chromosomes were observed between TR77 and 7182 than between TR77 and P. huashanica except for the 5th group chromosomes that had the lowest percentages of the same SNP loci between TR77 and 7182 (Table 3, Fig. 6a) and the chromosome $5 \mathrm{D}$ that had the highest percentage of the same SNP loci between TR77 and the alien parent $P$. huashanica, confirming the translocation occurred between wheat and P. huashanica on the chromosome homeologous 5. After comparison of the corresponding SNP positions in chromosome 5D between TR77 and its parent 7182 (Fig. 6b), we found an obvious boundary between Affymetrix SNP ID Affx-111,836,242 and Affx-111,527,395 located between $202.3 \mathrm{Mb}$ and $213.1 \mathrm{Mb}$ of $5 \mathrm{D}$ chromosome and assumed that translocation occurred in this interval.

\section{Agronomic traits and resistance to powdery mildew in TR77}

The agronomic traits were evaluated for TR77 and their parents (common wheat 7182, durum wheat Trs-372, $P$.

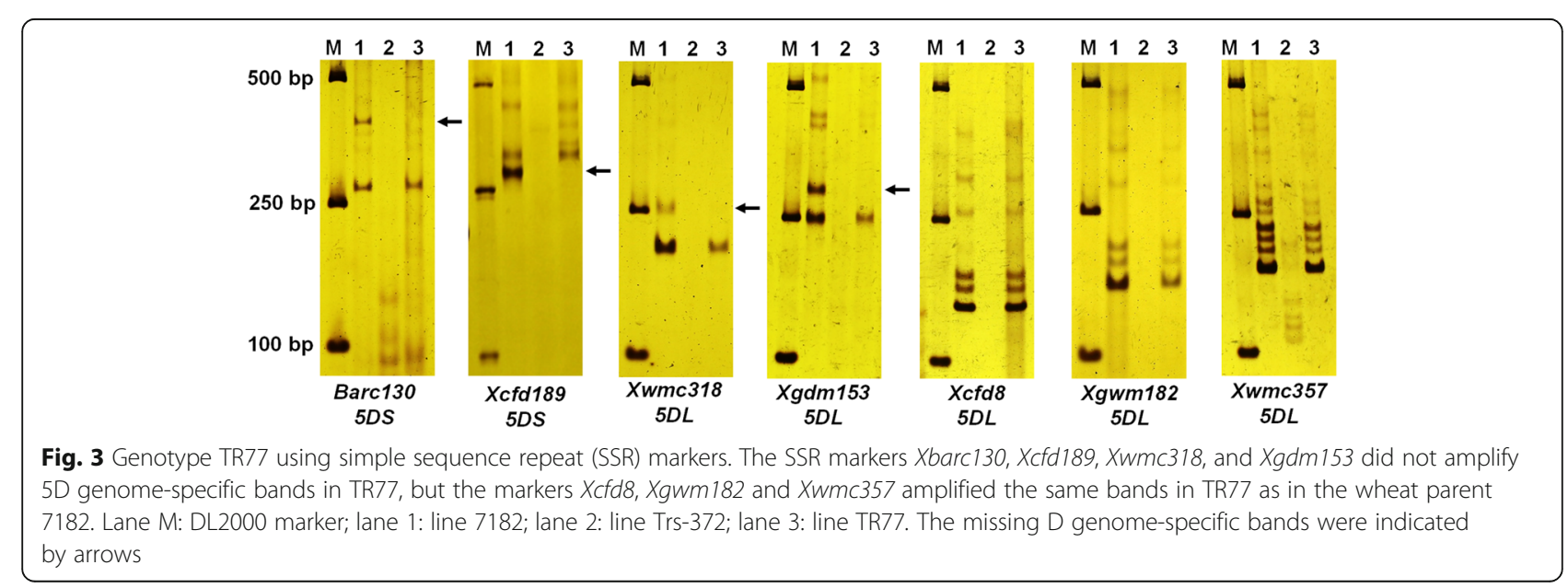




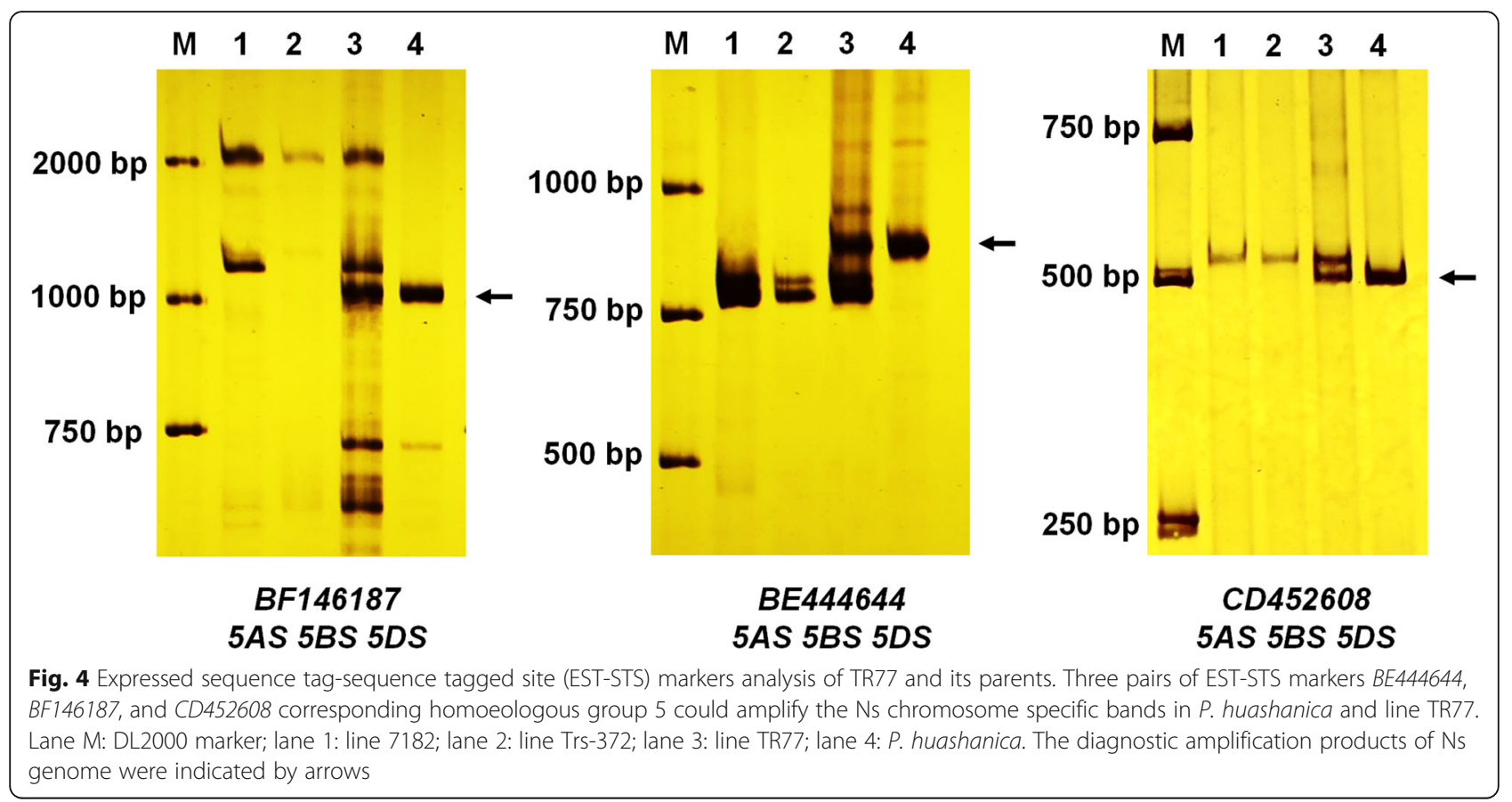

huashanica) (Table 4 and Fig. 7). TR77 exhibited completely different spike traits from its wheat parent. TR77 had conical and awnless spikes, taller plant, longer spikes, and more kernels per spike than its parents $(P<$ 0.01 ) between TR77 and its common wheat parent 7182 and Trs-372, indicating that addition of $5 \mathrm{Ns}$ chromosome of $P$. huashanica in TR77 increased spike length and spikelets number per spike, but also plant height.

The response of TR77 to powdery mildew infection was evaluated in both a growth chamber and a field. Control varieties, TR77, and its parents were grown under the same conditions to ensure accurate results. In the seedling age, susceptible mildew infection types (IT) were observed in Huixianhong (IT $=4)$, Mianyang 11 $(\mathrm{IT}=4)$, line $7182(\mathrm{IT}=3)$ and $\mathrm{Trs}-372(\mathrm{IT}=3)$, whereas $P$. huashanica $(\mathrm{IT}=0)$ and TR77 $(\mathrm{IT}=0$; ) were almost immune to powdery mildew (Fig. $7 \mathrm{~d}$ ). In the adult stage, the leaves of TR77 clearly had less spores than those of the control materials Mianyang 11 and Chinese Spring at the same positions on each plant (Fig. 7e).

\section{Discussion}

Wheat is a self-pollinating plant, and thus it may not evolve rapidly enough to meet the needs of modern humans. Modern wheat breeders usually create desired genetic variations by making crosses between breeding lines within bread wheat or using mutagenesis, transgenic approach, etc. [30]. However, for mutagenesis, controlling the direction of mutations is very difficult due to the random nature of this process, the large and complex genome of allopolyploid wheat and quantitative nature of the most target traits, and transformation genotype specificity limit transgenic wheat breeding to a few traits in a few genotypes [31], making crossing breeding the most widely used strategy for creation of new variations in wheat breeding. However, long time interbreeding has narrowed the wheat genetic diversity, and caused loss of many useful genes for disease resistance, and plant adaptation and productivity. Wide crosses between wheat and its relatives can introduce alien genetic materials into common wheat to generate desired genetic variations and create new germplasm, which is important for the sustainable development of new wheat cultivars [32]. P. huashanica is a wheat relative species with many desirable traits that are valuable for wheat improvement. Many wheat lines carrying genetic materials from $P$. huashanica show outstanding agronomic traits. For example, a wheat $-P$. huashanica 1 Ns disomic addition line exhibits increased storage of microelements in the seeds [10]. In addition, $2 \mathrm{Ns}, 3 \mathrm{Ns}, 4 \mathrm{Ns}$, and $5 \mathrm{Ns}$ disomic addition lines and a $2 \mathrm{Ns}(2 \mathrm{D})$ substitution line are resistant to stripe rust [13-16, 18]. A 6Ns disomic addition line and a small segment translocation line possess twin spikelets and more kernels per spike than its wheat parent [13, 21], and a 7Ns disomic addition line showed high resistance to leaf rust [12]. However, only a few wheat-P. huashanica translocation lines are available for breeding despite its importance. In the current study, we identified and characterized a novel wheat $-P$. huashanica translocation line that carries part of $P$. huashanica $5 \mathrm{Ns}$ chromosome and underwent dual reciprocal translocations in the 


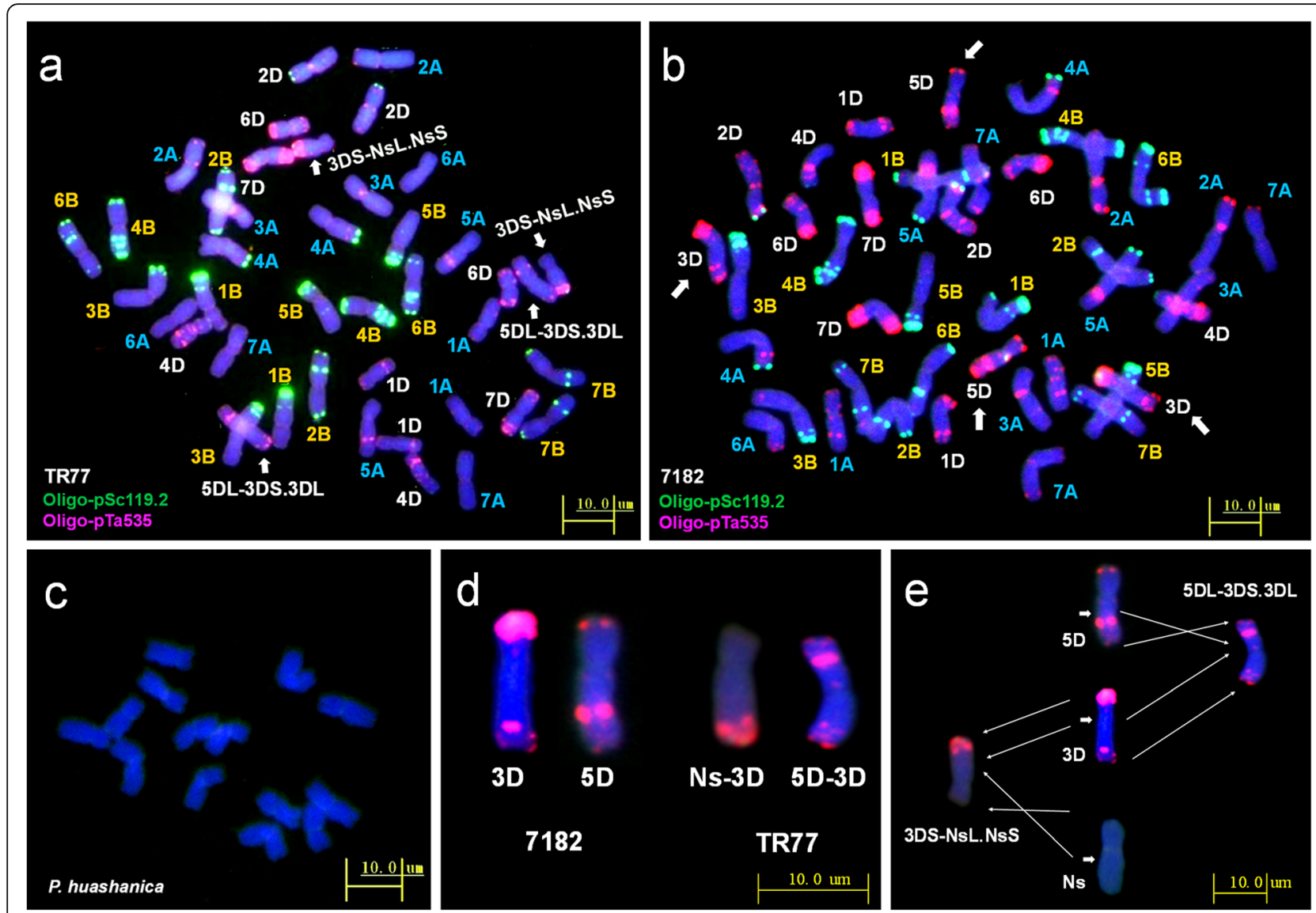

Fig. 5 Fluorescence in situ hybridization (FISH) analysis of TR77. Oligo-primers pSc119.2 (green) and pTa535 (red) were used as probes for wheat chromosomes during the mitosis metaphase in the root tip cells. a line TR77. b common wheat 7182. $\mathbf{c}$ P. huashanica. Wheat 3D and 5D chromosomes and chromosomes from two groups underwent double translocations to yield the 3DS-NsL.NsS and 5DL-3DS.3DL chromosomes as pointed by arrows. d Comparison of special chromosomes in common wheat 7182 and TR77. e Diagrammatic sketch of the two translocations, 3DS-NsL.NsS and 5DL-3DS.3DL, in TR77 to show breakage sites in each chromosome as pointed by arrows and chromosome rearrangement. Chromosomes were stained with 4,6-diamidino-2-phenylindole (blue)

wheat $3 \mathrm{D}$ and $5 \mathrm{D}$ chromosomes. This translocation line exhibited resistance to powdery mildew, an important wheat leave disease.

Whether alien chromosome segments can stably transmitted to their wheat offspring in a wheat background determines usefulness of the derived line in breeding [33]. In this study, the chromosomal compositions and behavior in the RTCs and PMCs of TR77 indicated 42 chromosomes with 21 bivalents in meiosis prophase I of TR77, with no lagging chromosomes during anaphase I. GISH analysis suggested that TR77 carries a pair of unique chromosomes that consist of chromosomal segments from both $P$. huashanica and wheat with the segment of P. huashanica from fifth homoeologous group (5Ns). The translocated $5 \mathrm{Ns}$ chromosome segment in TR77 paired normally in metaphase I and synchronized with the spliced wheat chromosome during the duplication and division phases, therefore, is stably inherited.
Genome specific DNA markers can be used to identify specific wheat chromosomes and determine homoeology of alien chromosome(s) with wheat. Du et al. [13] identified a wheat $-P$. huashanica $5 \mathrm{Ns}$ disomic addition line using EST-STS markers; Li et al. [19] identified a wheat$P$. huashanica 5Ns(5D) disomic substitution line using SSR, EST-STS, sequence characterized amplified region (SCAR) markers. In this study, we used wheat genomespecific SSRs and P. huashanica genome specific ESTSTSs to determine the chromosomal composition of TR77 and found that SSRs on 5DS and 5DL near the centromere amplified wheat genome-specific bands in parent 7182, but not in the translocation line TR77, whereas three EST-STSs on the fifth homoeologous group [13] amplified Ns genome-specific bands in both TR77 and $P$. huashanica. These results indicate that the short arm and partial long arm of wheat chromosome 5D close to the centromere were replaced by $P$. huashanica 
Table 3 Comparison of wheat $15 \mathrm{~K}$ SNP array data between TR77 and its parents

\begin{tabular}{|c|c|c|c|c|c|c|c|c|}
\hline Chromosome & $\begin{array}{l}\text { No. of } \\
\text { markers }\end{array}$ & $\begin{array}{l}\text { No. of valid } \\
\text { markers in } \\
\text { materials }\end{array}$ & $\begin{array}{l}\text { No. of same } \\
\text { markers (TR77 } \\
\text { vs 7182) }\end{array}$ & $\begin{array}{l}\text { Percentage of } \\
\text { same markers } \\
\text { (TR77 vs 7182) }\end{array}$ & $\begin{array}{l}\text { No. of same } \\
\text { markers (TR77 } \\
\text { vs Trs-372) }\end{array}$ & $\begin{array}{l}\text { Percentage of } \\
\text { same markers } \\
\text { (TR77 vs Trs-372) }\end{array}$ & $\begin{array}{l}\text { No. of same } \\
\text { markers (TR77 } \\
\text { vs P. huashanica) }\end{array}$ & $\begin{array}{l}\text { Percentage of } \\
\text { same markers } \\
\text { (TR77 vs } P \text {. } \\
\text { huashanica) }\end{array}$ \\
\hline $1 \mathrm{~A}$ & 607 & 408 & 211 & $52 \%$ & 304 & $75 \%$ & 23 & $6 \%$ \\
\hline 1B & 670 & 438 & 228 & $52 \%$ & 229 & $52 \%$ & 32 & $7 \%$ \\
\hline $1 D$ & 337 & 234 & 171 & $73 \%$ & - & - & 5 & $2 \%$ \\
\hline $2 \mathrm{~A}$ & 907 & 607 & 389 & $64 \%$ & 166 & $27 \%$ & 21 & $3 \%$ \\
\hline $2 B$ & 736 & 431 & 289 & $67 \%$ & 275 & $64 \%$ & 17 & $4 \%$ \\
\hline $2 \mathrm{D}$ & 597 & 404 & 353 & $87 \%$ & - & - & 8 & $2 \%$ \\
\hline $3 \mathrm{~A}$ & 594 & 391 & 294 & $75 \%$ & 149 & $38 \%$ & 27 & $7 \%$ \\
\hline $3 B$ & 997 & 616 & 398 & $65 \%$ & 432 & $70 \%$ & 20 & $3 \%$ \\
\hline $3 \mathrm{D}$ & 505 & 357 & 319 & $89 \%$ & - & - & 11 & $3 \%$ \\
\hline $4 \mathrm{~A}$ & 766 & 545 & 406 & $74 \%$ & 395 & $72 \%$ & 11 & $2 \%$ \\
\hline $4 B$ & 589 & 348 & 201 & $58 \%$ & 196 & $56 \%$ & 15 & $4 \%$ \\
\hline $4 \mathrm{D}$ & 248 & 165 & 148 & $90 \%$ & - & - & 12 & $7 \%$ \\
\hline $5 \mathrm{~A}$ & 690 & 414 & 166 & $40 \%$ & 203 & $49 \%$ & 32 & $8 \%$ \\
\hline $5 B$ & 763 & 462 & 222 & $48 \%$ & 286 & $62 \%$ & 22 & $5 \%$ \\
\hline $5 \mathrm{D}$ & 518 & 360 & 180 & $50 \%$ & - & - & 104 & $29 \%$ \\
\hline $6 \mathrm{~A}$ & 463 & 265 & 209 & $79 \%$ & 80 & $30 \%$ & 11 & $4 \%$ \\
\hline $6 B$ & 768 & 446 & 306 & $69 \%$ & 226 & $51 \%$ & 67 & $15 \%$ \\
\hline $6 \mathrm{D}$ & 404 & 279 & 260 & $93 \%$ & - & - & 11 & $4 \%$ \\
\hline $7 \mathrm{~A}$ & 735 & 490 & 326 & $67 \%$ & 320 & $65 \%$ & 26 & $5 \%$ \\
\hline $7 B$ & 640 & 404 & 354 & $88 \%$ & 228 & $56 \%$ & 12 & $3 \%$ \\
\hline 7D & 665 & 462 & 412 & $89 \%$ & - & - & 12 & $3 \%$ \\
\hline A genome & 4762 & 3120 & 2001 & $64 \%$ & 1617 & $52 \%$ & 151 & $5 \%$ \\
\hline B genome & 5163 & 3145 & 1998 & $64 \%$ & 1872 & $60 \%$ & 185 & $6 \%$ \\
\hline D genome & 3274 & 2261 & 1843 & $82 \%$ & - & - & 163 & $7 \%$ \\
\hline Total & 13,199 & 8526 & 5842 & $69 \%$ & 3489 & $41 \%$ & 499 & $6 \%$ \\
\hline
\end{tabular}

chromosome 5Ns. Many studies found that homoeologous chromosomes are remarkably conserved and any missing genetic content is usually complemented by corresponding homoeologous chromosomes [34-37]. In the H8911 $\times$ Trs- 372 cross, because Trs- 372 only carries $\mathrm{A}$ and $\mathrm{B}$ genomes, chromosome recombination mainly occurred between the $\mathrm{D}$ and Ns genomes, in particular, the reciprocal translocation between wheat $5 \mathrm{D}$ and $P$. huashanica $5 \mathrm{Ns}$ chromosomes that generated a new 5DL$5 \mathrm{NsL} \cdot 5 \mathrm{NsS}$ chromosome.

High-density SNP arrays can track sources of chromosomes from different species by comparing marker sequence identity between progeny and their donor parents. In the present study, comparison of a wheat 15 K SNP array data between TR77 and its parent 7182 confirmed that the D genome of TR77 was from 7182, whereas the $\mathrm{A}$ and $\mathrm{B}$ genomes were from chromosome recombination between wheat line 7182 and durum Trs372. TR77 genome had extremely low similarity with the genome of $P$. huashanica except for the chromosome 5D of TR77 that had high percentage of SNP loci in common with $P$. huashanica. Also, 5D of TR77 had lower percentage of SNP loci in common with its wheat parent 7182 compared with the other chromosomes. These results indicated the translocation most likely occurred in wheat chromosome 5D, which was supported by the results from previous studies of DNA markers and GISH. To determine the translocation site, we arranged all SNP markers on the chromosome 5D based on their physical locations in Chinese Spring reference genome and identified an obvious physical boundary between $202.3 \mathrm{Mb}$ and $213.1 \mathrm{Mb}$. The P. huashanica chromosomal segment was located before 202.3 $\mathrm{Mb}$ including the centromere region from $185.6 \mathrm{Mb}$ to $188.7 \mathrm{Mb}$ [38] and the common wheat (5DL) segment was located after $213.1 \mathrm{Mb}$ of the chromosome. Therefore, translocation break position in TR77 is near the centromere of wheat 5D chromosome. 


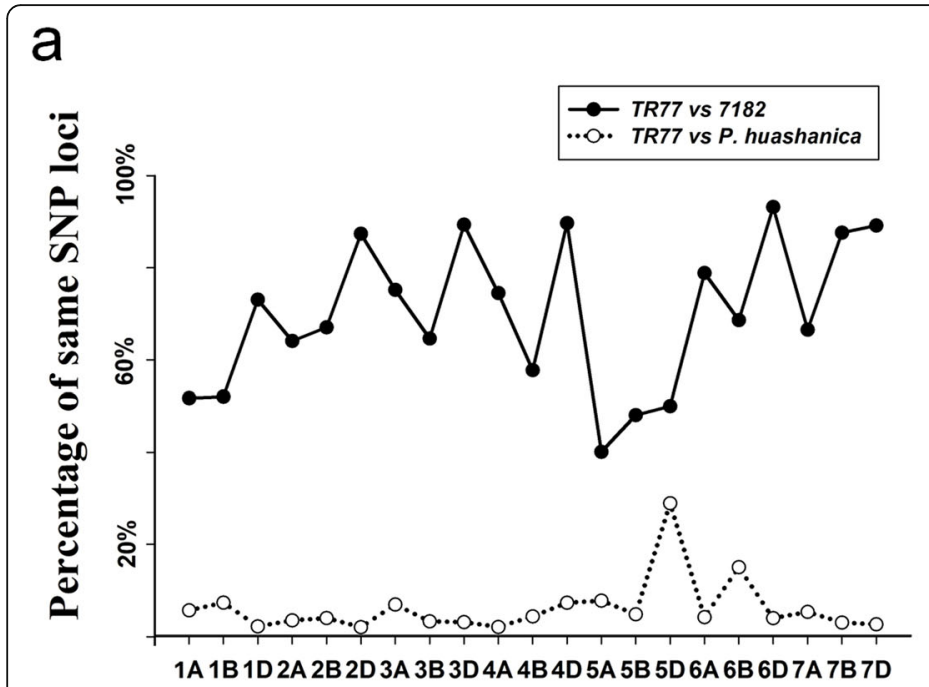

Position of markers on wheat chromosomes b

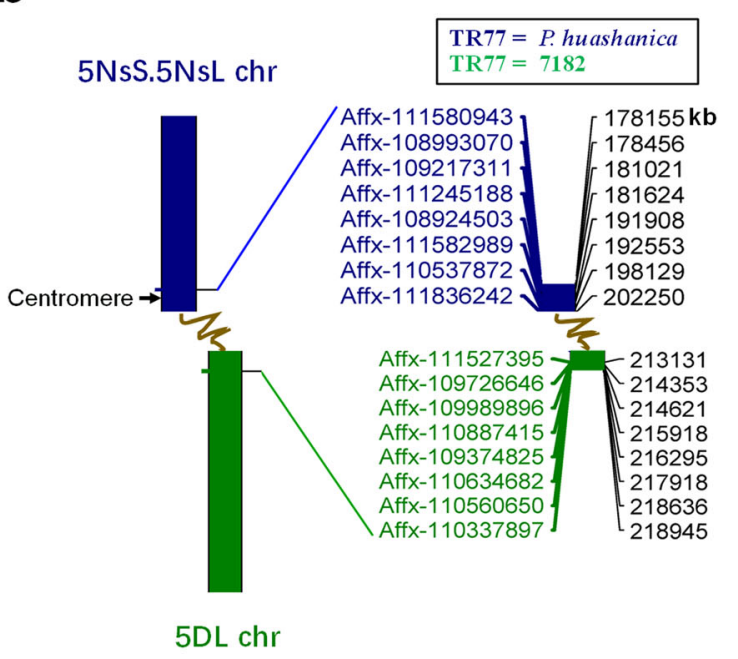

Fig. 6 Chromosomal composition of TR77 analyzed using a wheat $15 \mathrm{~K}$ SNP array. a Difference in the 5D chromosomes according to the percentages of the same SNP loci. b The same genotype SNPs were arranged in the 5D chromosome between TR77 and its parents. An obvious boundary was determined between Affymetrix SNP ID Affx-111,836,242 and Affx-111,527,395, where the physical location was between 202.3 Mb and $213.1 \mathrm{Mb}$

The FISH probes, Oligo-pSc119.2 and Oligo-pTa535, can accurately distinguish all 42 common wheat chromosomes based on the standard FISH karyotypes in mitotic cells $[39,40]$. Many wheat-derived lines including substitution and translocation lines have been identified using this method [41-43]. In addition, structural changes in wheat chromosomes can be detected by FISH. For example, FISH was used to confirm a reciprocal translocation of the 5BL-7BS chromosome in a wheat variety Humai 15 and a reciprocal translocation of the 4BL-6AL chromosome in a wheat variety Mingxian 169 [44]. In this study, however, FISH did not detect 5DL$5 \mathrm{NsL} .5 \mathrm{NsS}$ chromosome, therefore, $5 \mathrm{NsL} \cdot 5 \mathrm{NsS}$ might undergo another translocation within wheat chromosome, which is confirmed by FISH identification of two translocations, 3DS-5NsL.5NsS and 5DL-3DS.3DL in TR77. A previous FISH karyotype study on line 7182 did not find translocation between wheat chromosomes 3D and 5D [29]. Thus, the first translocation might have occurred between the wheat 5D and P. huashanica $5 \mathrm{Ns}$ chromosomes to yield chromosome 5DL-5NsL.5NsS as transition, and then a reciprocal translocation occurred between the wheat $3 \mathrm{D}$ and $5 \mathrm{DL}-5 \mathrm{NsL} \cdot 5 \mathrm{NsS}$ chromosomes that produced 3DS-5NsL.5NsS and 5DL-3DS.3DL in TR77. During the two translocation events, TR77 lost its short arm and partial long arm of wheat 5D chromosome and acquired short arm (5NsS) and partial long arm (5NsL) of $P$. huashanica chromosome 5. Therefore, TR77 is a wheat $-P$. huashanica translocation line with dual translocations of $3 \mathrm{DS}-5 \mathrm{NsL} \cdot 5 \mathrm{NsS}$ and 5DL3DS.3DL. The $5 \mathrm{NsL} \cdot 5 \mathrm{NsS}$ segment was combined with the wheat 3D chromosome via inter-chromosomal recombination. The fifth group of chromosomes in wheat carries the Ph1 gene that controls the cross ability among species [45]. A previous study found that the wheat 5BL chromosome contained a subtelomeric insertion from wheat chromosome 3A [46]. Also a wheat line carrying T5BS.7BS, T5BL.7BL and T5AS.3BS chromosomes was identified using FISH technology [47]. Those studies support the possible reciprocal translocations between wheat chromosomes $5 \mathrm{D}$ and $3 \mathrm{D}$ occurred in this study.

Table 4 Agronomic traits of TR77, its parents, P. huashanica, common wheat 7182, and durum wheat Trs-372

\begin{tabular}{|c|c|c|c|c|c|c|c|}
\hline Material & Plant height $(\mathrm{cm})$ & Tiller number & $\begin{array}{l}\text { Spike length } \\
(\mathrm{cm})\end{array}$ & $\begin{array}{l}\text { Spikelets } \\
\text { per spike }\end{array}$ & Kernels per spike & $\begin{array}{l}\text { Thousand kernel } \\
\text { weight (g) }\end{array}$ & Awn type \\
\hline P. huashanica & $63.5 \pm 2.2 \mathrm{Dd}$ & clump & $8.26 \pm 0.96 \mathrm{BCbc}$ & $16 \pm 2 \mathrm{BCb}$ & $42 \pm 6 C c$ & $3.44 \pm 0.11 \mathrm{Cc}$ & Short awn \\
\hline 7182 & $83.8 \pm 1.4 \mathrm{Bb}$ & $9 \pm 2 \mathrm{Ab}$ & $9.05 \pm 0.57 \mathrm{Bb}$ & $18 \pm 3 \mathrm{ABa}$ & $54 \pm 4 \mathrm{Bb}$ & $37.69 \pm 0.74 \mathrm{Aa}$ & Long awn \\
\hline Trs-372 & $76.3 \pm 2.9 \mathrm{Cc}$ & $10 \pm 2 \mathrm{Aab}$ & $7.91 \pm 0.33 \mathrm{Cc}$ & $14 \pm 2 \mathrm{Cb}$ & $43 \pm 2 C c$ & $35.52 \pm 0.6 \mathrm{Bb}$ & Long awn \\
\hline TR77 & $92.6 \pm 2.3 \mathrm{Aa}$ & $12 \pm 3 \mathrm{Aa}$ & $13.05 \pm 0.77 \mathrm{Aa}$ & $19 \pm 3 \mathrm{Aa}$ & $67 \pm 5 \mathrm{Aa}$ & $37.56 \pm 0.69 \mathrm{Aa}$ & Awnless \\
\hline
\end{tabular}




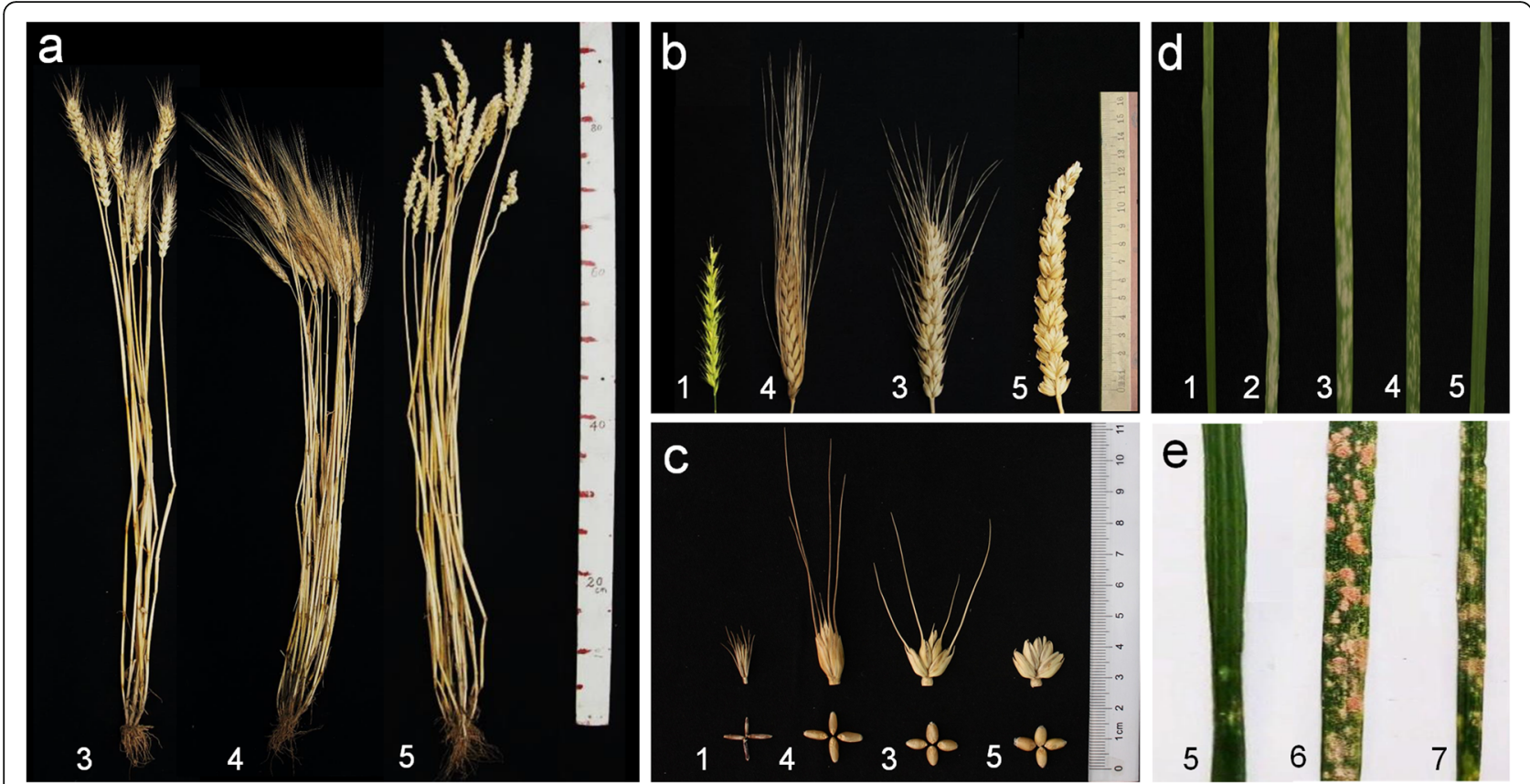

Fig. 7 Agronomic traits and powdery mildew resistance in the translocation line TR77, its parents, and controls. a Plants. b Spikes. c Kernels. d Symptoms in response to inoculation with a mixture of Bgt race E09 races in the seedling stage. e Symptoms in response to powdery mildew after natural infection in the adult stage. The samples in the Figure are (1) P. huashanica; (2) Huixianhong; (3) 7182; (4) Trs-372; (5) TR77; (6) Mianyang 11 and (7) Chinese Spring

Powdery mildew resistance genes from wild relatives of wheat are usually durable with broad-spectrum of resistance [48]. Pm21 was transferred from Haynaldia villosa 6VS to wheat and a highly powdery mildew resistant 6VS/6AL translocation line is still effective and widely used in production [27]. The current study showed that TR77 was near immune to powdery mildew in both seedling and adult ages, although its two wheat parents exhibited susceptible symptoms, indicating that powdery mildew resistance trait in TR77 was from $P$. huashanica. So, the identification of TR77 filled a gap where no wheat-P. huashanica derived lines had excellent powdery mildew resistance before. Agronomic traits are important criteria for assessing the value of a line carrying alien chromosome segments. TR77 had larger spikes and more kernels per spike than its wheat parents, thereby has potential to increase yield. Crossing TR77 with locally adapted varieties can transfer these desired agronomic traits to new wheat cultivars. Line TR77 exhibited many excellent characteristics, which meant these excellent genes most possible introduced from $P$. huashanica because of chromosomal exchange and recombination between wheat and $P$. huashanica. The replaced segment were entire short arm and partial long arm of the chromosome close to centromere which was generally considered to contain few expressible genes. Therefore, the gene(s) that control awn length may be located in chromosome
5DS and raise powdery mildew resistance and spikes development may be located in chromosome $5 \mathrm{NsS}$.

\section{Conclusion}

In this study, TR77 was identified as a new wheat- $P$. huashanica T3DS-5NsL.5NsS and T5DL-3DS.3DL dual translocation line with larger spikes, more kernels per spike, and better powdery mildew resistance than their wheat parents. Molecular, cytogenetic and phenotypic analyses on TR77 determined its chromosomal composition, translocation position and inheritance stability as well as its superior agronomic performance. TR77 can be an important germplasm for improving powdery mildew resistance and yields in wheat breeding, and for dual translocation research.

\section{Methods}

\section{Plant materials}

The plant materials include one line of Psathyrostachys huashanica Keng $(2 n=14$, NsNs) from Huashan Mountains, Shaanxi province, China, one common wheat (Triticum aestivum L.) line $7182(2 n=42$, AABBDD $)$, one durum (Triticum durum) line Trs-372 $(2 n=28$, AABB), and the wheat $-P$. huashanica translocation line TR77 obtained from the $F_{7}$ progeny of the cross between line Trs372 and a wheat $-P$. huashanica derived line H8911(2n= 49, AABBDDNs). The heptaploid line H8911 was created 
by the wide cross between $P$. huashanica and line 7182 via artificial embryo rescue and backcross [8]. Common wheat varieties Huixianhong, Mianyang 11, and Chinese Spring were used as controls for assessing disease resistance.

The wheat wild related material $P$. huashanica was identified and collected by Shuyang Chen and Langran $\mathrm{Xu}$ who were the first researchers to do hybridization between common wheat and $P$. huashanica in China in 1980s [8]. The wheat materials were collected from The National Wheat Improvement Center of China and the wheat-P. huashanica derived lines were developed by our lab. All materials were deposited and planted in College of Agronomy, Northwest A\&F University, China. The collection and treatment of materials for this experiment complied Wild Plants Protection Regulation of China. Extraction and purification of genomic DNA from leaf tissues used the standard Cetyltrimethylammonium Ammonium Bromide method [49].

\section{Cytological analysis}

The appropriate stages for sampling were length of roots to $1.5 \mathrm{~cm}$ and young panicles to $5 \mathrm{~cm}$ [50]. The cut roots were immediately soaked in ice-water mixture for 12 $20 \mathrm{~h}$ before transferring to Carnoy's fixative fluid I for $24 \mathrm{~h}$, and finally stored at $-20^{\circ} \mathrm{C}$. Young panicles at appropriate stage were treated with Carnoy's fixative fluid II for $24 \mathrm{~h}$ and then stored under refrigeration. The root apical meristem was digested in $2 \%$ cellulase plus $1 \%$ pectinase at $37^{\circ} \mathrm{C}$ for more than $50 \mathrm{~min}$ before scattering in acetic acid. The chromosomes in root tip cell were observed under a microscope at 400 times magnification (OLYMPUS BH2, Japan). Anthers were stained with $1 \%$ acetocarmine and gently broke for cytological observations. The slides with good split phases were dried and marked for the following experiments. Microscopic observations were the first step in order to identify the chromosomal number and behavior in TR77. In this process, each plant was numbered to ensure root and spike from the same plants.

\section{DNA marker analysis}

A total of 384 pairs of SSR primers [51,52] were selected from 21 wheat chromosomes to determine the translocated wheat chromosomes in TR77. In addition, to identify the homoeologous group of the introduced alien chromosomes in TR77, 87 pairs of EST-STS markers (https://wheat.pw.usda.gov/SNP/new/pcr_primers.shtml) were selected from seven wheat homoeologous groups that had homoeology with corresponding chromosomes of $P$. huashanica. PCR assays were conducted following Doğrar et al. [53] and an ABI PRISM 3730 DNA Analyzer (Applied Biosystems, USA) was adopted for separation of the PCR products. The data of SSR markers were scored by GeneMarker V1.97 (Soft Genetics LLC, USA).

\section{In-situ hybridization}

For the GISH analysis, Mixed the P. huashanica genomic DNA and DiG-Nick Translation Mix (Roche, Germany) in a ratio of two to one at $15^{\circ} \mathrm{C}$ for $1.5 \mathrm{~h}$ as labeling probes following Wetzel et al. [54] and Zhao et al. [55]. The addition of Anti-Digoxigenin-Fluorescein mix (Roche, Germany) made the probes visualize after hybridization. The wheat chromosomes were dyed using Vectashield H1300 (Vector Laboratories, USA). In the FISH analysis, the match of Oligo-pSc119.2 (6FAM-5'CCGTTTTGTG GACTATTACT CACCGC TTTG GGGTCCCATA GCTAT) and Oligo-pTa535-1 (Tamra-5'AAAAACTTGA CGCACGTCAC GTACAA ATTG GACAAACTCT TTCGGAGTAT CAGGGTTC) were used to give insight into the chromosomal composition of TR77. After hybridizing with wheat chromosomes, the standard karyotype of the two Oligo-probes could be seen [28, 39]. FISH experiment was conducted as described by Patokar et al. [56] and Lang et al. [57]. The signals were observed and captured using OLYMPUS BX60 fluorescence microscope with a color camera (Penguin, Japan) at 400 times magnification.

\section{Characterization of wheat-P. huashanica translocation using wheat $15 \mathrm{~K}$ SNP arrays}

High-quality genomic DNA of TR77 and its parents was used to hybrid with Illumina wheat $15 \mathrm{~K} \mathrm{SNP}$ arrays for loci difference scanning in China Golden Marker Biotechnology Company (Beijing, China). The array contained 13,199 SNP loci, which were dispersed in all 21 wheat chromosomes. The total valid number of SNP loci divided by the loci number that had the same genotype in a chromosome between materials was calculated as the percentage of the same markers in each chromosome. Data analysis used SigmaPlot V12.5 (SYSTAT software, USA) and chromosomal map was drew using MapChart V2.32 (Wageningen University \& Research, The Netherlands).

\section{Characterization of agronomic traits and powdery mildew resistance}

P. huashanica, TR77 and its wheat parents were evaluated in the field for seven agronomic traits, i.e. spike length, plant height, spikelet number, tiller number, kernel number, thousand-kernel weight and self-fertility. The mean values over five plants were collected in two successive years as repeats to ensure data accuracy.

The powdery mildew resistance was evaluated at the seedling stage in a growth chamber. The powdery mildew Bgt race E09 was used for inoculation with wheat variety Huixianhong as a susceptible control. The pathogen spores were dusted onto wheat leaves at twoleaf stage and incubated at $22^{\circ} \mathrm{C}$ and $70 \%$ humidity for $15 \mathrm{~d}$. the adult plant powdery mildew resistance was 
evaluated in a field in Sichuan province, China using Mianyang 11 as the susceptible control. The field usually had a high incidence of powdery mildew and thus tested plants were infected naturally. The reactions to powdery mildew were assessed following Sheng [58] and An [59]. In brief, wheat responses to infection were scored using infection type (IT) in a $0-4$ IT scale at the seedling stage, in which IT $=0,0 ; 1,2,3$ and 4 denoting immune, nearly immune, highly resistant, moderately resistant, moderately susceptible and susceptible respectively. In the adult stage, the plant responses to infection were recorded as the percentage of the powdery mildew spores covered the total area of the leaves at the same position on each plant [60].

\section{Supplementary information}

Supplementary information accompanies this paper at https://doi.org/10. 1186/s12870-020-02366-8

Additional file 1. Figure S1 TR77-Molecular markers uncropped images.

Additional file 2. Table S1 TR77-SSR Analysis.

Additional file 3. Table S2 TR77-15K SNP array.

\section{Abbreviations}

EST-STS: Expressed sequence tag-sequence tagged site; FISH: Fluorescence in situ hybridization; GISH: Genomic in situ hybridization; IT: Immunity type; P. huashanica: Psathyrostachys huashanica Keng; PMC: Pollen mother cell; SCAR: Sequence characterized amplified region; SNP: Single nucleotide polymorphism; SSR: Simple sequence repeat; RTC: Root tip cell

\section{Acknowledgements}

None.

\section{Authors' contributions}

$J \mathrm{~L}, \mathrm{JZ}$ and $Z Y$ conducted experiments. $\mathrm{LZ}$ and $\mathrm{ML}$ analyzed data. XNC and GB contributed new reagents and analytical tools. JW and QY contributed new methods or models. JZ and XHC conceived and designed research. JL and JZ wrote and GB edited the paper. All authors read and approved the final version of the manuscript.

\section{Funding}

This research is partially supported by the National Science Foundation of China (31571650 and 31771785), National Key Research and Development Program of China (2017YFD0100701), Key Projects in Shaanxi Provincial Agricultural Field (2018ZDXM-NY-006) and Basic Research Projects of the Natural Science Foundation of Shaanxi Province (2015JM3095). The funders Jixin Zhao and Xinhong Chen conceived and designed this research. The funding bodies didn't play a role in the design of the study and collection, analysis, and interpretation of data and in writing the manuscript.

\section{Availability of data and materials}

All related plant materials are available and comply Wild Plants Protection Regulation of China. The datasets supporting the conclusions of this article are included within the article and its supplementary files.

\section{Ethics approval and consent to participate}

Not applicable.

\section{Consent for publication}

Not applicable.

\section{Competing interests}

The authors declare that they have no competing interests.

\section{Author details}

TShaanxi Key Laboratory of Plant Genetic Engineering Breeding, College of Agronomy, Northwest A\&F University, Yangling 712100, Shaanxi, China. ${ }^{2}$ College of Life Science, Northwest A\&F University, Yangling 712100, Shaanxi, China. ${ }^{3}$ USDA, Hard Winter Wheat Genetics Research Unit, 4008

Throckmorton Hall, Manhattan, KS 66506, USA. ${ }^{4}$ School of Life Science and Technology, University of Electronic Science and Technology of China, Chengdu 610054, Sichuan, China.

Received: 2 November 2019 Accepted: 26 March 2020

Published online: 15 April 2020

\section{References}

1. Fischer RA, Edmeades G. Breeding and cereal yield Progress. Crop Sci. 2010; 50(1):S85-98.

2. Mujeeb-Kazi A, Kazi AG, Dundas I, Rasheed A, Ogbonnaya F, Kishii M, Bonnett D, Wang RRC, Xu S, Chen P, et al. Chapter four-genetic diversity for wheat improvement as a conduit to food security. In: Sparks DL, editor. Advances in Agronomy, vol. 122: Academic Press; 2013. p. 179-257.

3. Tester M, Langridge P. Breeding technologies to increase crop production in a changing world. Science. 2010;327(5967):818.

4. Han HM, Bai L, Su JJ, Zhang JP, Song LQ, Gao AN, Yang XM, Li XQ, Liu WH, $\mathrm{Li}$ LH. Genetic rearrangements of six wheat-Agropyron cristatum 6P addition lines revealed by molecular markers. PLoS One. 2014:9(3):e91066.

5. Sharma P, Sheikh I, Kumar S, Verma SK, Kumar R, Vyas P, Dhaliwal HS. Precise transfers of genes for high grain iron and zinc from wheat-Aegilops substitution lines into wheat through pollen irradiation. Mol Breed. 2018; 38(6):81.

6. Z Zhang QP, Li Q, Wang X, Wang HY, Lang SP, Wang YN, Wang SL, Chen PD, Liu DJ. Development and characterization of a Triticum aestivum-Haynaldia villosa translocation line T4VS $4 \mathrm{DL}$ conferring resistance to wheat spindle streak mosaic virus. Euphytica. 2005;145(3):317-20.

7. Rabinovich SV. Importance of wheat-rye translocations for breeding modern cultivar of Triticum aestivum L. Euphytica. 1998;100(1):323-40.

8. Chen SY. The hybridization between Triticum aestivum and Psathyrostachys huashanica. Acta Genet Sin. 1991;18(6):508-12

9. Fu J, Wang MN, Zhao JX, Chen SY, Hou WS, Yang QH. Studies on cytogenetics and utilization of wheat-Psathyrostachys huashanica medium material H8911 with resistance to wheat take-all fungus. Acta Botan BorealiOcciden Sin. 2003;23(12):2157-62.

10. Zhao JX, Ji WQ, Wu J, Chen XH, Cheng XN, Wang JW, Pang YH, Liu SH, Yang $\mathrm{QH}$. Development and identification of a wheat-Psathyrostachys huashanica addition line carrying HMW-GS, LMW-GS and gliadin genes. Genet Resour Crop Evol. 2010:57(3):387-94.

11. Li Q, Huang J, Hou L, Liu P, Jing J, Wang B, Kang Z. Genetic and molecular mapping of stripe rust resistance gene in wheat-Psathyrostachys huashanica translocation line H9020-1-6-8-3. Plant Dis. 2012;96(10):1482-7.

12. Du WL, Wang J, Wang LM, Zhang J, Chen XH, Zhao JX, Yang QH, Wu J. Development and characterization of a Psathyrostachys huashanica Keng 7Ns chromosome addition line with leaf rust resistance. PLoS One. 2012; 8(8):e70879.

13. Du WL, Wang J, Lu M, Sun SG, Chen XH, Zhao JX, Yang QH, Wu J. Molecular cytogenetic identification of a wheat-Psathyrostachys huashanica Keng 5Ns disomic addition line with stripe rust resistance. Mol Breed. 2013;31(4):87988

14. Du W, Wang J, Wang L, Wu J, Zhao J, Liu S, Yang Q, Chen X. Molecular characterization of a wheat-Psathyrostachys huashanica Keng 2Ns disomic addition line with resistance to stripe rust. Mol Gen Genomics. 2014;289(5): 735-43.

15. Du WL, Wang J, Lu M, Sun SG, Chen XH, Zhao JX, Yang QH, Wu J. Characterization of a wheat-Psathyrostachys huashanica Keng 4Ns disomic addition line for enhanced tiller numbers and stripe rust resistance. Planta. 2014;239(1):97-105.

16. Du WL, Wang J, Pang YH, Wang LM, Wu J, Zhao JX, Yang QH, Chen XH. Isolation and characterization of a wheat-Psathyrostachys huashanica 'Keng'3Ns disomic addition line with resistance to stripe rust. Genome. 2014:57(1):37-44.

17. Du WL, Wang J, Pang YH, Wu J, Zhao JX, Liu SH, Yang QH, Chen XH. Development and application of PCR markers specific to the $1 \mathrm{Ns}$ chromosome of Psathyrostachys huashanica Keng with leaf rust resistance. Euphytica. 2014;200(2):207-20. 
18. Du WL, Zhao JX, Wang J, Wang LM, Wu J, Yang QH, Liu SH, Chen XH. Cytogenetic and molecular marker-based characterization of a wheatPsathyrostachys huashanica Keng 2Ns(2D) substitution line. Plant Mol Biol Report. 2015;33(3):414-23.

19. Li JC, Yao XN, Yang Z, Cheng XN, Yuan FP, Liu Y, Wu J, Yang QH, Zhao JX, Chen $\mathrm{XH}$. Molecular cytogenetic characterization of a novel wheat-Psathyrostachys huashanica Keng 5Ns (5D) disomic substitution line with stripe rust resistance. Mol Breed. 2019;39(7):109.

20. Cao Z, Deng Z, Wang M, Wang X, Jing J, Zhang X, Shang H, Li Z. Inheritance and molecular mapping of an alien stripe-rust resistance gene from a wheatPsathyrostachys huashanica translocation line. Plant Sci. 2008;174(5):544-9.

21. Kang HY, Zhang Z, Xu LL, Qi WL, Tang Y, Wang H, Zhu W, Li DY, Zeng J, Wang Y. Characterization of wheat-Psathyrostachys huashanica small segment translocation line with enhanced kernels per spike and stripe rust resistance. Genome. 2016;59(4): 221-9.

22. Wang Y, Yu K, Xie Q, Kang H, Lin L, Fan X, Sha L, Zhang H, Zhou Y. The 3Ns chromosome of Psathyrostachys huashanica carries the gene(s) underlying wheat stripe rust resistance. Cytogenetic Genome Res. 2011;134(2):136-43.

23. Ma DF, Zhou XL, Hou L, Bai YB, Li Q, Wang HG, Tang MS, Jing JX. Genetic analysis and molecular mapping of a stripe rust resistance gene derived from Psathynrostachys huashanica Keng in wheat line H9014-121-5-5-9. Mol Breed. 2013;32(2):365-72.

24. An DG, Zheng Q, Zhou YL, Ma PT, Lv ZL, Li LH, Li B, Luo QL, Xu HX, Xu YF. Molecular cytogenetic characterization of a new wheat-rye $4 \mathrm{R}$ chromosome translocation line resistant to powdery mildew. Chromosom Res. 2013;21(4):419-32.

25. Wang YJ, Quan W, Peng NN, Wang CY, Yang XF, Liu XL, Zhang H, Chen CH, Ji WQ. Molecular cytogenetic identification of a wheat- Aegilops geniculata Roth $7 \mathrm{M}^{9}$ disomic addition line with powdery mildew resistance. Mol Breed. 2016:36:40.

26. Zhan HX, Xiaojun Z, Li GR, Pan ZH, Hu J, Li X, Qiao LY, Jia JQ, Guo HJ, Chang ZJ, et al. Molecular characterization of a new wheat-Thinopyrum intermedium translocation line with resistance to powdery mildew and stripe rust. Int J Mol Sci. 2015;16:2162-73.

27. Cao $A Z$, Xing $L P$, Wang $X Y$, Yang $X M$, Wang $W$, Sun $Y L$, Qian $C$, Ni JL, Chen YP, Liu DJ, et al. Serine/threonine kinase gene Stpk-V, a key member of powdery mildew resistance gene Pm21, confers powdery mildew resistance in wheat. Proc Natl Acad Sci. 2011;108:7727-32.

28. Annamaria S, Linc G, Molnar-Lang M, Graner A. Fluorescence in situ hybridization polymorphism using two repetitive DNA clones in different cultivars of wheat. Plant Breed. 2003;122:396-400.

29. Yang XF, Wang CY, Li X, Chen CH, Tian ZR, Wang YJ, Ji WQ. Development and molecular cytogenetic identification of a novel wheat-Leymus mollis Im\#7Ns (7D) disomic substitution line with stripe rust resistance. PLoS One. 2015;10(10):e0140227.

30. Smith EL, Mac Key J, Qualset C. Conventional methods of wheat breeding. In: Genetic Improvement in Yield of Wheat. America: Crop Science Society of America and American Society of Agronomy; 1986.

31. Bajaj YPS, Gosal SS. Biotechnology of Wheat Improvement. In: YPS B, editor. Crops I. Berlin: Springer Berlin Heidelberg; 1986. p. 3-38.

32. Li Z, Li B, Tong Y. The contribution of distant hybridization with decaploid Agropyron elongatum to wheat improvement in China. J Gen Genomics. 2008;35(8):451-6.

33. Cifuentes $M$, Benavente E. Wheat-alien metaphase I pairing of individual wheat genomes and D genome chromosomes in interspecific hybrids between Triticum aestivum L. and Aegilops geniculata Roth. Theor Appl Genet. 2009;119(5):805-13.

34. Li CZ, Huang HQ, Yin FY, Wang ZY, Peng YK, Xie CJ, Liu ZY, Sun QX, Yang ZM. The effect of Haynaldia villosa $\vee$ chromosome on the mitochondrial proteome of wheat- $\mathrm{H}$. villosa chromosome substitution line and translocation line. J Mol Cell Biol. 2008;41(2):150-4

35. Ren TH, Chen F, Yan BJ, Zhang HQ, Ren ZL. Genetic diversity of wheat-rye 1BL.1RS translocation lines derived from different wheat and rye sources. Euphytica. 2012;183(2):133-46.

36. Danilova TV, Friebe B, Gill BS, Poland J, Jackson E. Chromosome rearrangements caused by double monosomy in wheat-barley group-7 substitution lines. Cytogenet Genome Res. 2018;154(1):45-55.

37. Du XY, Ma X, Min JZ, Zhang XC, Jia ZZ. Development of a wheat-Aegilops searsii substitution line with positively affecting Chinese steamed bread quality. Breed Sci. 2018;68(2):289-93.

38. Appels R, Eversole K, Stein N, Feuillet C, Keller B, Rogers J, Pozniak CJ, Choulet F, Distelfeld A, Poland J, et al. Shifting the limits in wheat research and breeding using a fully annotated reference genome. Science. 2018; 361(6403):eaar7191.

39. Tang ZX, Yang Z, Fu SL. Oligonucleotides replacing the roles of repetitive sequences pAs1, pSc119.2, pTa-535, pTa71, CCS1, and pAWRC.1 for FISH analysis. J Appl Genet. 2014;55(3):313-8.

40. Qi WL, Tang Y, Zhu W, Li DY, Diao CD, Xu LL, Zeng J, Wang Y, Fan X, Sha $L N$, et al. Molecular cytogenetic characterization of a new wheat-rye 1BL-1RS translocation line expressing superior stripe rust resistance and enhanced grain yield. Planta. 2016;244(2):405-16.

41. Zhu C, Wang YZ, Chen CH, Wang CY, Zhang A, Peng NN, Wang YJ, Zhang H, Liu $X L$, Ji WQ. Molecular cytogenetic identification of a wheat-Thinopyrum ponticum substitution line with stripe rust resistance. Genome. 2017;60(10):860-7.

42. Wang J, Liu YL, Su HD, Guo XR, Han FP. Centromere structure and function analysis in wheat-rye translocation lines. Plant J. 2017;91(2):199-207.

43. Wang HJ, Zhang HJ, Li B, Yu ZH, Li GR, Zhang J, Yang ZJ. Molecular cytogenetic characterization of new wheat- Dasypyrum Breviaristatum introgression lines for improving grain quality of wheat. Frontiers Plant Sci. 2018;9:365.

44. Yuan FP, Zeng QD, Wu JH, Wang QL, Yang ZJ, Liang BP, Kang ZS, Chen XH, Han DJ. QTL mapping and validation of adult plant resistance to stripe rust in chinese wheat landrace humai 15. Front Plant Sci. 2018:9:968.

45. Sidhu G, Rustgi S, Shafqat M, Wettstein D, Gill K. Fine structure mapping of a gene-rich region of wheat carrying Ph1, a suppressor of crossing over between homoeologous chromosomes. Proc Natl Acad Sci U S A. 2008;105: 5815-20.

46. Griffiths S, Sharp R, Foote TN, Bertin I, Wanous M, Reader S, Colas I, Moore G. Molecular characterization of $\mathrm{Ph} 1$ as a major chromosome pairing locus in polyploid wheat. Nature. 2006;439(7077):749-52.

47. Yang MY, Yang ZJ, Yang WY, Yang EN. Development and identification of new wheat varieties (lines) with multiple translocation chromosomes via cytogenetic method. J Triticeae Crops. 2018;38(2):127-33.

48. Chen SG, Wang JX. Screening wild relatives of wheat for disease reisistance. Sci Agric Sin. 1990;23(1):54-9.

49. Doyle JJ, Doyle JL. A rapid DNA isolation procedure for small quantities of fresh leaf tissue. Phytochem Bull. 1987;19:11-5

50. Danilova TV, Friebe B, Gill BS. Single-copy gene fluorescence in situ hybridization and genome analysis: Acc-2 loci mark evolutionary chromosomal rearrangements in wheat. Chromosoma. 2012;121(6):597-611.

51. Pestsova E, Ganal MW, Röder MS. Isolation and mapping of microsatellite markers specific for the D genome of bread wheat. Genome. 2000;43(4): 689-97.

52. Somers DJ, Isaac P, Edwards K. A high-density microsatellite consensus map for bread wheat (Triticum aestivum L.). Theor Appl Genet. 2004;109(6):1105-14.

53. DOĞRAR N, Akkaya MS. Optimization of PCR amplification of wheat simple sequence repeat DNA markers. Turk J Biol. 2001;25(2):153-8.

54. Wetzel JB, Rayburn AL. Use of fluorescence genomic in situ hybridization $(\mathrm{GISH})$ to detect the presence of alien chromatin in wheat lines differing in nuclear DNA content. Cytometry. 2000;41:36-40.

55. Zhao JX, Du WL, Wu J, Cheng XN, Gao Y, Pang YH, Chen XH, Liu SH, Yang $\mathrm{QH}, \mathrm{Fu}$ J. Development and identification of a wheat-Leymus mollis multiple alien substitution line. Euphytica. 2013:190(1):45-52.

56. Patokar C, Sepsi A, Schwarzacher T, Kishii M, Heslop-Harrison JS. Molecular cytogenetic characterization of novel wheat-Thinopyrum bessarabicum recombinant lines carrying intercalary translocations. Chromosoma. 2016; 125(1):163-72.

57. Lang T, Li GR, Wang HJ, Yu ZH, Chen QH, Yang EN, Fu SL, Tang ZX, Yang ZJ. Physical location of tandem repeats in the wheat genome and application for chromosome identification. Planta. 2019;249(3):663-75.

58. Sheng BQ. Grades of resistance to powdery mildew classified by different phenotypes of response in the seeding stage of wheat. Plant Prot. 1988;1:49.

59. An DG, Ma PT, Zheng Q, Fu SL, Li LH, Han FP, Han GH, Wang J, Xu YF, Jin $Y L$, et al. Development and molecular cytogenetic identification of a new wheat-rye $4 R$ chromosome disomic addition line with resistances to powdery mildew, stripe rust and sharp eyespot. Theor Appl Genet. 2019; 132(1):257-72

60. Liu SX, Griffey C, Maroof S. Identification of molecular markers associated with adult plant resistance to powdery mildew in common wheat cultivar massey. Crop Sci. 2001;41:1268-75.

\section{Publisher's Note}

Springer Nature remains neutral with regard to jurisdictional claims in published maps and institutional affiliations. 